\title{
Research and Design on Electro-Optic Obscuring System of High Pressure Water Mist
}

\author{
Yuejun He \\ Shenzhen Polytechnic, Xili Lake, Nanshan District, Shenzhen, Guangdong, China \\ 386160724@qq.com
}

Keywords: high pressure, water mist, obscuring system, nozzle

Abstract. The paper first elaborate the composition principle of the electro-optic obscuring system of high-pressure water mist. Then, the design principle of the system's core component - pulsed eddy current nozzle is introduced. Finally, the obscuring effect of the system is verified through the infrared thermal imaging experiment. The experimental results show that high-pressure water mist generated by the system can greatly reduce the infrared signature of objects to be obscured with significant electro-optic obscuring effect. The system can be used for homeland defense with high practical value.

\section{Introduction}

High-pressure water mist generated by high-pressure water jet technology is a new broad-spectrum electro-optic jamming material, which has a strong obscuring and jamming effect on infrared light, laser, radar and millimeter waves ${ }^{[1-3]}$. Ordinary water after slight filtration can serve as a jamming medium with low cost and pollution. High-pressure water mist can be used for electro-optic obscuring of various ground objects and marine objects like ships and warships to guard against electro-optic precision guided weapons from enemies, which will play an increasingly important role in the field of homeland security and defense. However, the existing types of water mist obscuring devices mainly use the technology of low-pressure fogging or conventional water jet fogging with disadvantages of large droplet size, short hang time, high settlement velocity, small obscured area, large water consumption and high water cleanness, so they are not practical nor suitable to obscure for military purpose.

\section{The principle and characteristics of water mist obscuring system}

To overcome the disadvantages of the existing water mist obscuring devices, a high-pressure water mist obscuring system based on pulsed eddy current nozzle ${ }^{[4-6]}$ is designed in this paper. The block diagram of the system is shown in Fig.1. This system uses pulsed eddy current water jet technology ,automatic control technology, hydraulic drive and control technology as well as multiple disciplines of hydroacoustics, fluid resonance and fluid elasticity. Its working principle is as follows: the system first uses the energy storage device to store the energy provided by the power source (three-phase asynchronous motor), and then uninterruptedly delivers it to the water filtered through strainer. The filtered water is appropriately pressurized via the high-pressure pump in the high-pressure water generator and flows into the nozzle. When the system works, the oscillator in the nozzle produces high-frequency pulsation and self-excited oscillation in the fluid by applying an pulsed eddy current excitation source to convert the continuous water flow into oscillating pulsed water flow. Then, the oscillating pulsed water rotates at high speed via the rotary core in the nozzle to generate large centrifugal force. Finally, the water is ejected from the nozzle forming high-energy pulsed eddy current water mist to obscure electro-optic objects. In this system, the application of the high-speed rotating eddy current technology helps overcome the surface tension of water molecules and fragment the water into fine water mist with long hang time in the air so as to improve the efficiency and electro-optic obscuring effect of the water mist obscuring system. 


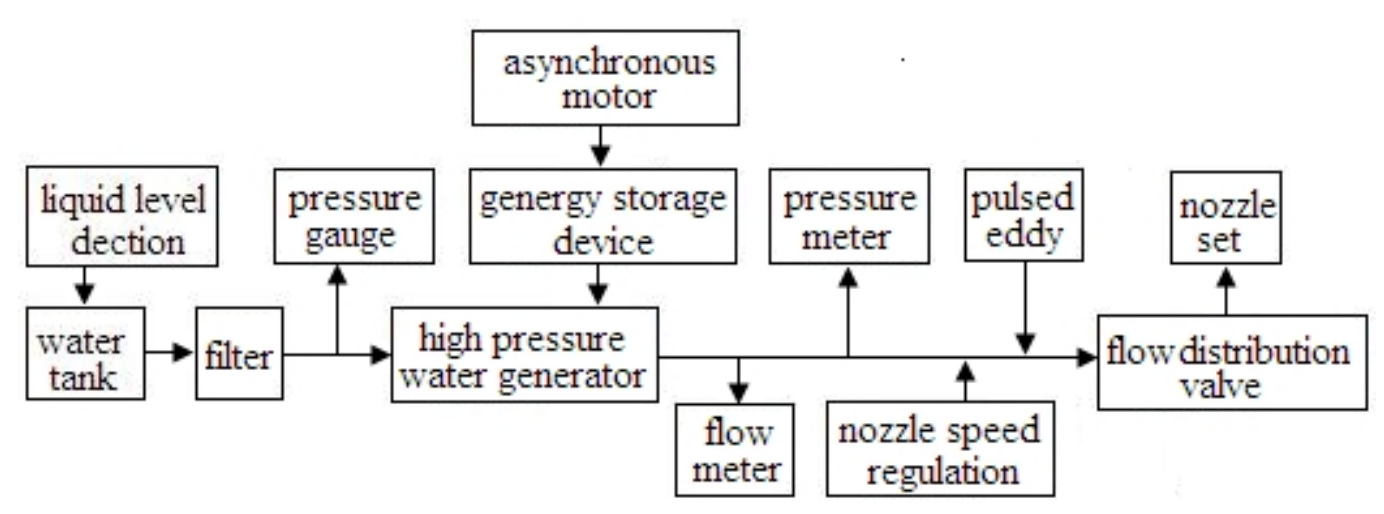

Fig.1 the block diagram of the electro-optic obscuring system

\section{Overall design of Water Mist Obscuring System}

The water mist obscuring system uses automatic coordinated control technology and is easy to operate. The working principle of the system is shown in Fig.1. A liquid level controller is installed at the upper part of the tank in the system for water level monitoring and alarm. A filter is connected to the outlet of the tank with a strainer and filtration class of $20 \mathrm{um}$.

The main parameters of the system's power unit are shown in Table 1. Two groups of rotatory pulsed eddy current nozzle are connected in parallel to the outlet of the system's high-pressure water generator. The system is equipped with flow meters and pressure meters to monitor flow and water pressure. The nozzles are independently developed and designed by the research team of this project. The nozzle shape design drawing is shown in Fig.2. The real nozzle is shown in Fig.3. A fan is fixed on the mount of the nozzle and equipped with blades. Each nozzle is equipped with a protective cover. The pipe of the nozzle is bent $35^{\circ}$ so that the high-pressure water mist sprayed out of the nozzle can avoid the protective cover.

The nozzle is a high precision component of the system and its parts are made of titanium alloy and high-strength stainless steel with resistance to corrosion and high pressure. To improve the water mist effect, the system sprays by means of multiple nozzles in parallel, which facilitates operator to flexibly arrange the system according to field requirements. The flow distribution valve in the system is used to control the flow rate of high-pressure water flowing into different nozzles. The water pressure can be adjusted by adjusting the speed of the three-phase asynchronous motor. The system applies pulsed eddy current to the nozzle to obtain a high-frequency eddy water jet so that the water molecules are sufficiently atomized when the high-pressure water is ejected from the outlet to form fine droplets. Fig. 4 shows the structure of the pulsed eddy current nozzle. Operator adjusts the speed of the rotatory device by adjusting the viscosity and concentration of the lubricating oil in the rotatory device of the nozzle to obtain optimal electro-optic obscuring effect of water mist.

\section{Structural design of water mist nozzle}

The nozzle is the core component of the water mist obscuring system to form high-pressure water mist. The design of the nozzle directly affects the electro-optic obscuring effect of the system. The ultrasonic wave generated by the ultrasonic generator in the system converts the electric pulse into standing wave by the high frequency vibration of the transducer, which is converted from electric energy into mechanical energy in the pulse generating cavity 1 of Fig.4 thus to change ordinary water jet into high-frequency pulsed jet. When the high-frequency pulsed jet flows through the eddy current resonator 4 in the nozzle, the high-frequency pulsed jet is converted into pulsed eddy current jet due to the swirling cavitation. Meanwhile, the nozzle rotates around the rotation axis in planar motion at high speed with the help of high-speed bearings 2 under the action of swirling water, thereby converting the pulsed eddy current jet into high-speed rotating pulsed eddy current jet. Finally, the 
high-speed rotating pulsed eddy current jet is ejected from the nozzle at supersonic speed forming high-speed and high-energy

Table 1. the main parameters of the system's power unit

\begin{tabular}{|l|l|}
\hline Parameter & Numerical value \\
\hline Motor power & $7.5 \mathrm{KW}$ \\
\hline $\begin{array}{l}\text { Rated voltage } \\
\text { of Motor }\end{array}$ & $380 \mathrm{~V}$ \\
\hline $\begin{array}{l}\text { Rated speed } \\
\text { of Motor }\end{array}$ & $1440 \mathrm{RPM}$ \\
\hline $\begin{array}{l}\text { Maximum Pump } \\
\text { Pressure }\end{array}$ & $50 \mathrm{MPa}$ \\
\hline $\begin{array}{l}\text { Maximum pump } \\
\text { flow }\end{array}$ & $15.5 \mathrm{~L} / \mathrm{M}$ \\
\hline
\end{tabular}

pulsed eddy current water mist. Operator can adjust the rotation speed of the nozzle by adding lubricating oil of different viscosity and concentration into the rotating mechanism through the oil nozzle 3 to improve the electro-optic obscuring effect of water mist.

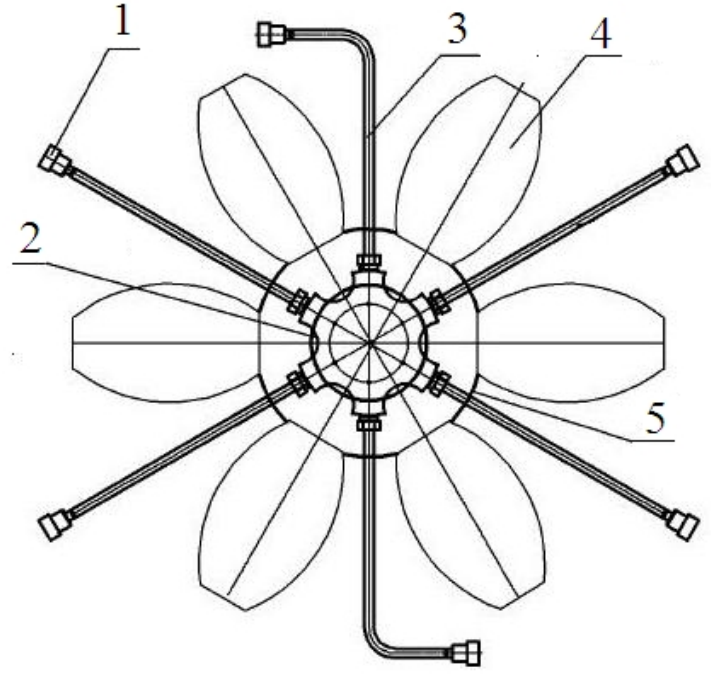

1. nozzle seat 2.nozzle mounting body 3.connecting pipe 4.fan blade 5.fan body

Fig.2 The nozzle shape design

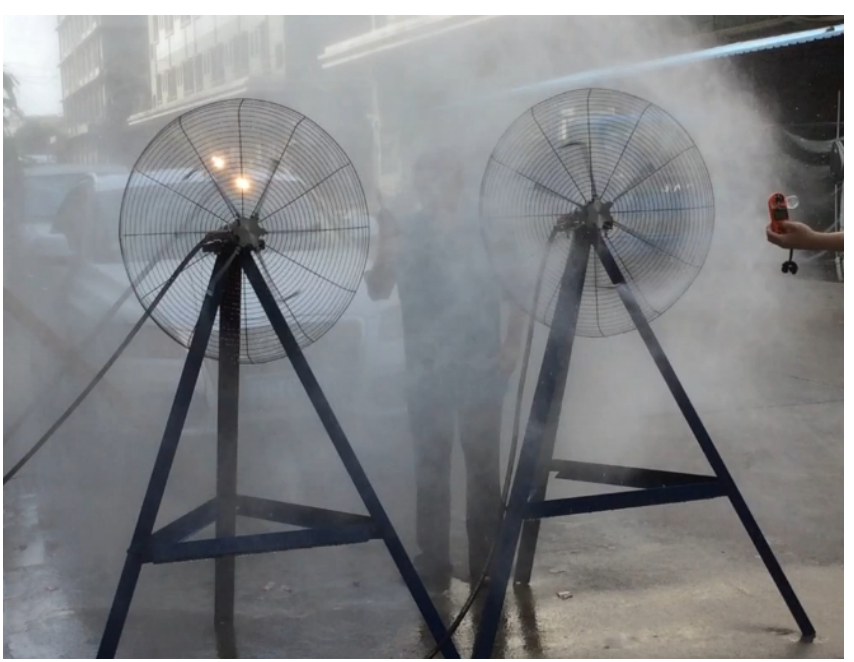

Fig.3 The real nozzle

\section{Experimental Study of Water Mist Obscuring System}

To test the effect of the high-pressure water mist obscuring system on the attenuation of multi-spectrum visible and infrared light, and analyze the influence and rule of main control parameters of eddy-current nozzle on electro-optic obscuring effect, the infrared thermal imaging experiment of high-pressure water mist was carried out for comparison.Fig.6 shows the layout of the experimental equipment .

A minivan was used as the research subject to perform with the infrared thermal imaging experiment before and after applying water mist for comparison. The water mist obscuring system was located 4 meters from the object (minivan) to be obscured, between the object and the test instrument like thermal imager. The test instrument was 21 meters away from the object to be obscured. The experiment was carried out in the absence of light at night.

The experimental process is as follows. The experimenter sat in the minivan, started the minvan and lit a cigarette. About 12 minutes later, experimental targets(include the minivan, cigarette and the 
experimenter) is in thermal equilibrium. From infrared thermal image of Fig.6, the experimental targets are clearly infrared.At this point, the water mist obscuring system is used to apply water mist, after applying water mist for 3 seconds, as shown in infrared thermal image of Fig.7.The infrared features of experimenter, cigarette and engine have begun to blur and their infrared contours are not known. 12s after the water mist was applied, the infrared signatures of the experiment objects were disappeared in the infrared thermal image of Fig.8. 25s after the water mist dispersed, the infrared signatures of the experiment objects were still not distinct in the infrared thermal image.

It shows that water mist obscuring system played an important role in the scattering, absorption of infrared radiation of the objects. The infrared photoelectric shielding effect of the water mist obscuring system is excellent.

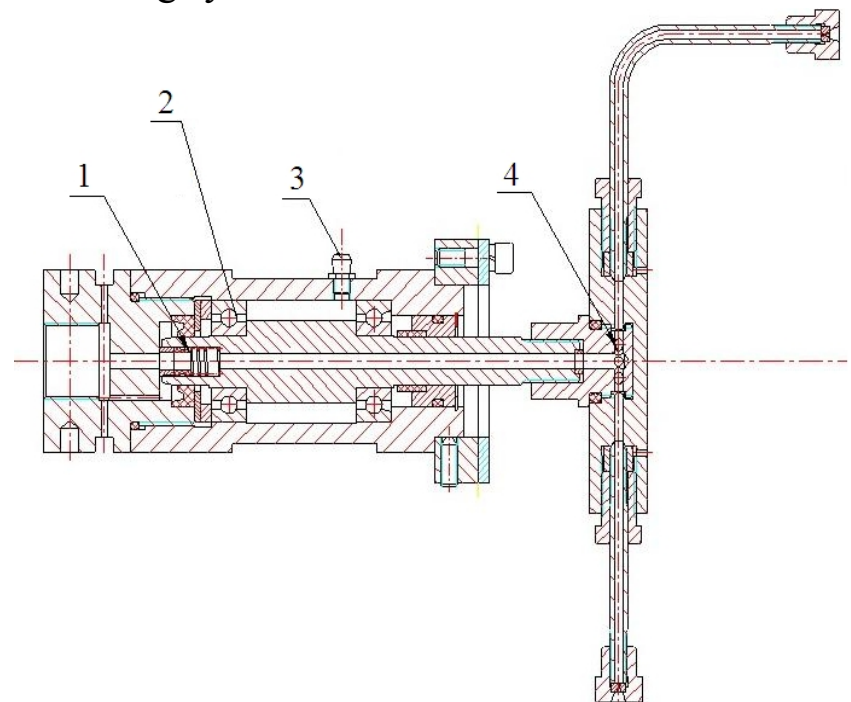

1.pulse generator cavity 2 .high speed bearing 3.oil nozzle 4.vortex resonator

Fig.4 The structure of the nozzle

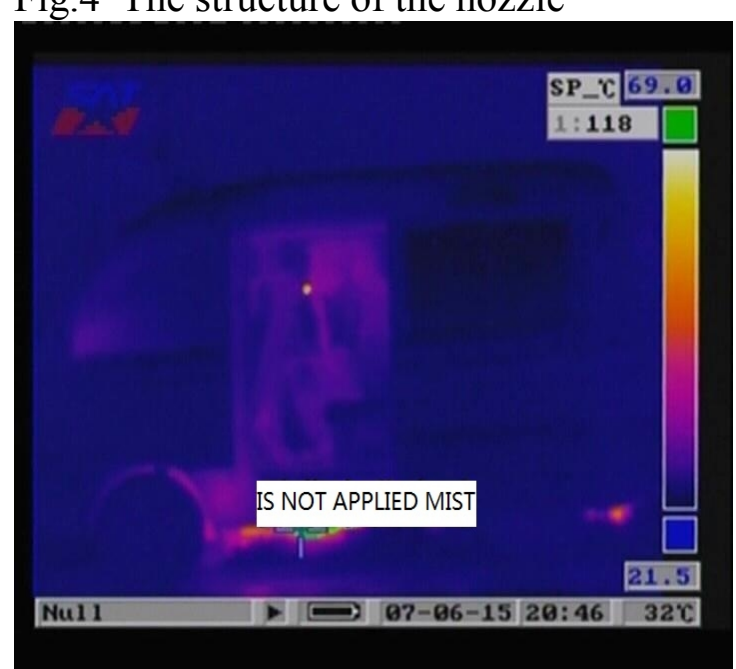

Fig.6 Thermal image

(before applying water mist)

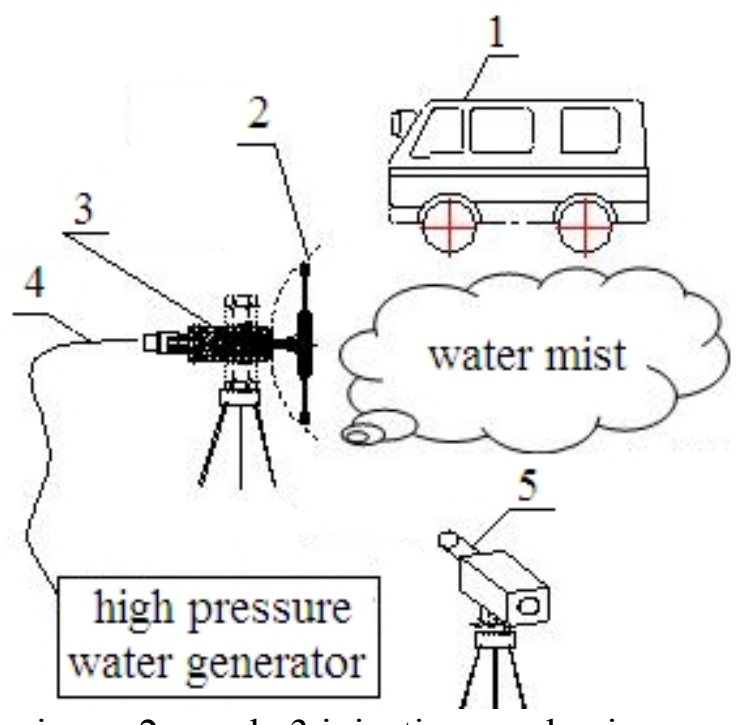

1.minvan 2.nozzle 3.injection mechanism 4.high pressure hose 5.red thermal imager

Fig.5 The layout of the experiment equipment

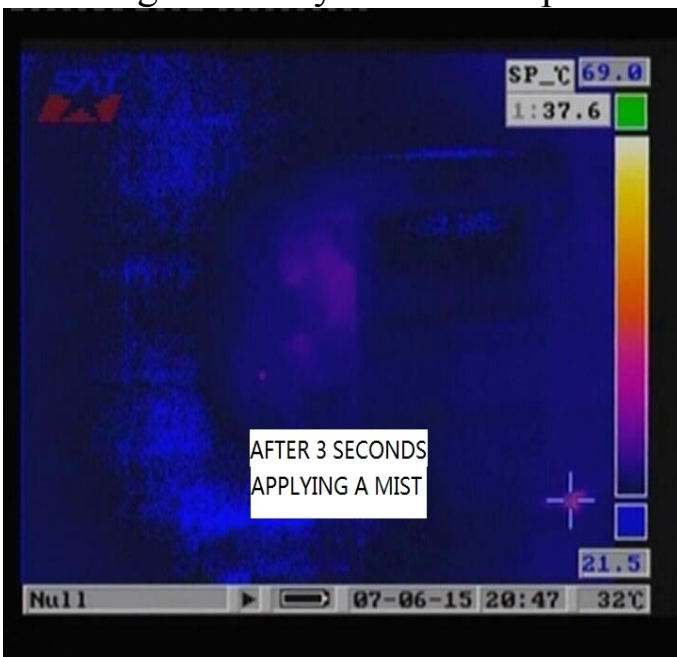

Fig.7 Thermal image

(when water was applied for $6 \mathrm{~s}$ )

\section{Conclusions}

1) The obscuring system uses the fogging technology of high-pressure and high-speed rotatory pulsed eddy current with Low water consumption and pollution. The water mist produced by rotatory nozzle is fully atomized into fine droplets and the high pressure water mist generated by the system has a strong attenuation effect on infrared signature.

2) The high-pressure water mist ejected from the double nozzle of the obscuring system can greatly reduce the infrared signature of objects to be obscured in a very short time, blending the objects and 
background with significant electro-optic obscuring effect and practical application in the field of homeland defense.

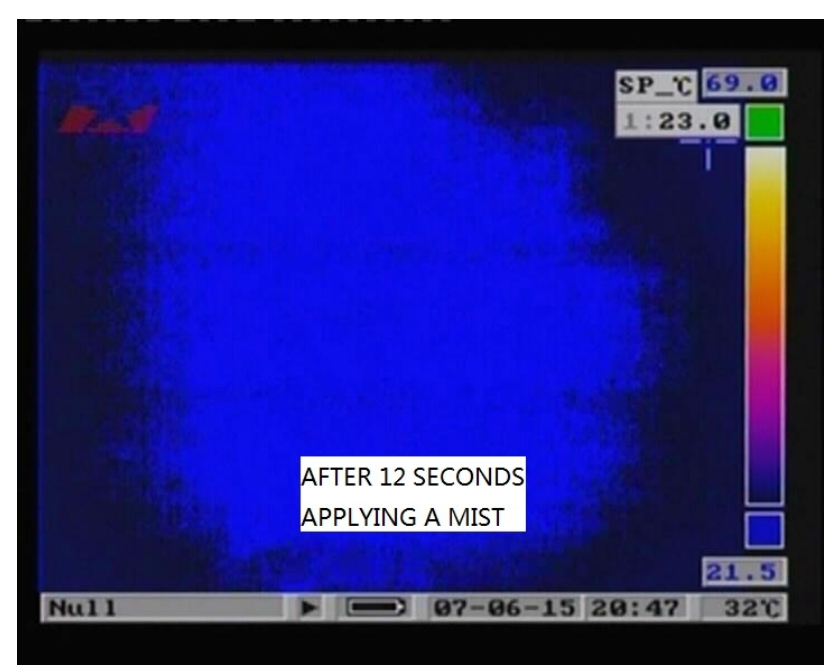

Fig. 8 Thermal imge

(when water was applied for 12s)

\section{References}

[1] Hu Biru,Wu Wenjian,Dai Mengyan,et al .Study on Property of Infrared Obscure of Artificial Fog[J].J Infrared millin waves,2006,25(2):131-134

[2] Chen Zhongwei,Liang Xingang,Zhang Lingjiang, et al .Investigation of Spray on Temperature Drop and Infrared Stealth of Water Surface Object[J],J Infrared Millim Waves,2010,29(5):342-346

[3]Du Yongcheng, Yang Li. Study on optimal extinction radius of water fog particles based on phase function and extinction factor [J]. Acta Optical Sinica , 2010, 30 (8): 2459 -2463

[4] Shen Zhong-hou, Li Gen-sheng, et al. New jet theory and prospects of application in drilling engineering[ A].Proc. of 13th World Petroleum Congress[ C]. Buenos Aires, 1991. 397-405.

[5] Liao Z F, Tang C L. Theoretical analysis and experimental study of the self-excited oscillation pulse jet device [ R]. Proceedings 4th U S Water jet Conference. 1987, 27 34

[6] Jonson V E Jr, Conn A F, et al. Cavitating and structured jets for mechanical bits to increase drilling rate[J]. Trans ASME Journal of Energy Resources Technolo-gy, 1984, 106: 282-294. 